\title{
The Effect of Plant Secondary Metabolites on Lipid Peroxidation and Eicosanoid Pathway
}

\author{
Neda Mimica-Dukić, Nataša Simin, Emilija Svirčev, \\ Dejan Orčić, Ivana Beara, Marija Lesjak and Biljana Božin
}

Additional information is available at the end of the chapter

http://dx.doi.org/10.5772/48193

\section{Introduction}

Inflammation, free radical damage and oxidative stress have become major health issues in recent years and the subject of plenty of research. These processes are implicated in cancer [1], cardiovascular diseases [2], multiple sclerosis [3], diabetes mellitus [4], Alzheimer's and Parkinson's diseases [5], rheumatoid arthritis [6], premature aging [7] and almost any other degenerative condition. Reactive oxygen species (ROS), which are involved in these physiological functional changes, are often either by-products of the normal cellular processes or are formed by action of exogenous factors - xenobiotics, ionizing radiation, stress, pathogens etc. Overproduction of ROS leads to oxidative stress, with biomolecules, including lipids, proteins and nucleic acids undergoing oxidative alterations.

\subsection{Lipid peroxidation}

Lipid peroxidation (LP) - oxidative degradation of polyunsaturated fatty acids caused by ROS - is responsible for degradation of membrane lipids resulting in cell damage and formation of many toxic products. LP is a free radical chain reaction where three major steps - the initiation, propagation and termination - can be recognised (Figure 1.).

In initiation phase, highly reactive hydroxyl radical, formed in Fenton reaction, abstracts hydrogen atom in $\alpha$ position relative to the polyunsaturated fatty acid double bond. This results in the formation of fatty acid radical, highly unstable, short-lived intermediate that stabilises by abstracting hydrogen from another chemical species, or reacts with triplet oxygen to generate different radical species, including fatty acid peroxyl radical. In the termination step peroxyl radicals transform into nonradical compounds - hydrocarbons, aldehydes, alcohols, volatile ketones and lipid polymers, some of which are harmful (Figure 1.) $[8,9]$. 


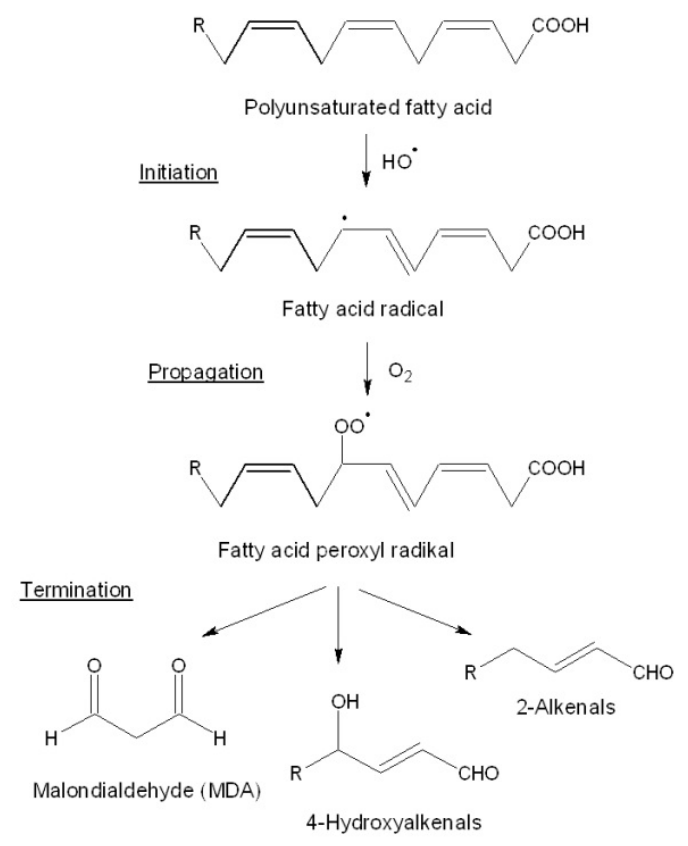

Figure 1. Steps in lipid peroxidation process

Organism uses a number of endogenous antioxidants, such as glutathione, $\alpha$-lipoic acid, coenzyme Q10, bilirubin and antioxidant enzymes (glutathione peroxidase, catalase, superoxide dismutase), to protect itself from oxidative stress. When they are insufficient, it becomes necessary to introduce exogenous antioxidants. Most of these compounds are primarily taken into the body by food and are predominantly of herbal origin (phenolics, carotenoids, terpenoids and vitamins - ascorbic acid and tocopherol). Herbal antioxidants exhibit their activity through a wide variety of mechanisms, such as inhibition of oxidising enzymes, chelation of transition metals, transfer of hydrogen or single electron to radicals, singlet oxygen deactivation, or enzymatic detoxification of ROS [8]. They can stop LP process in either initiation or propagation step. Herbal antioxidants have become subjects of growing interest and targets of numerous scientific research. However, enormous number of plant species is still waiting to be investigated in this manner and explored as potential medical drugs or dietary supplements.

\subsection{Eicosanoid pathway}

In addition to direct detrimental effects on biomolecules and cellular structures, lipid peroxidation is also involved in biosynthesis of eicosanoids - arachidonic acid metabolites serving as inflammatory mediators. Arachidonic acid, released from phospholipids by action of phospholipase A, can be converted into these products by three different pathways: cyclooxygenase, leading to the formation of prostanoids (prostaglandins and thromboxanes), lipoxygenase, where leukotrienes and certain mono-, di- and trihydroxy 
acids are synthesised, and epoxygenase pathway, which includes cytochrome P-450 and gives epoxides as final products [10].

Cyclooxygenase (COX), key enzyme in cyclooxygenase pathway (Figure 2.), exists in three forms, COX-1, COX-2, and recently discovered COX-3. Despite the differences in structure, localisation and regulation, reactions catalysed by COX isoforms follow the same mechanism. All of them transform arachidonic acid into prostanoids: prostaglandins ( $\mathrm{PGH}_{2}$, PGE2, PGF $2 \alpha$ ), thromboxanes (TXA2, TXB 2 ), and 12(S)-hydroxyheptadeca-5Z,8E,10E-trienoic acid (12(S)-HHT) as a co-product.

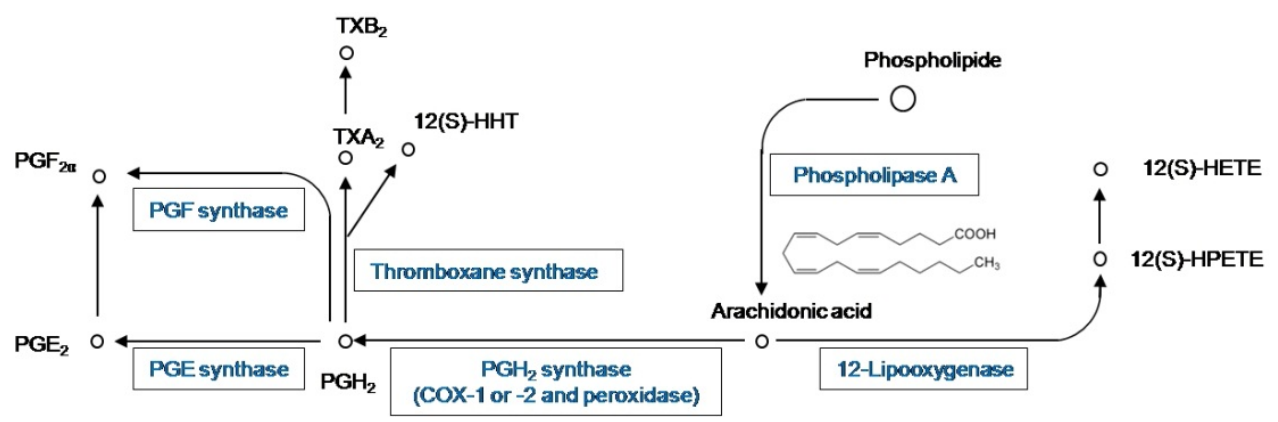

Figure 2. COX and 12-LOX branches of eicosanoid pathway

COX-catalysed transformation of arachidonic acid starts with tyrosyl radical generation through $\mathrm{Tyr}^{385}$ oxidation by heme in COX active site. Formed tyrosyl radical abstracts hydrogen from C-13 of arachidonic acid. In subsequent steps, free electron migration, reaction with oxygen yielding peroxyl radical, and cyclisation reactions give PGG2 which is converted to $\mathrm{PGH}_{2}$ through the action of peroxidase (Px) (Figure 3.) [11].

COX-1 is expressed constitutively in different tissues, blood monocytes and platelets, and is involved in normal cellular homeostasis. In contrast, COX-2 may be induced by a series of pro-inflammatory stimuli and its role in the progress of inflammation, fever and pain has been known [12]. Furthermore, COX-2 has been targeted in many cancers including: colon cancer, colorectal cancer, breast cancer, gliomas, prostate cancer, esophageal carcinoma, pancreatic cancer, lung carcinoma, gastric carcinoma, ovarian cancer, Kaposi's sarcoma and melanoma [13].

In lipoxygenase branch of arachidonic acid metabolism, there are three types of lipoxygenases, termed 5-, 12- and 15-lipoxygenase. In 12-lipooxigenase (12-LOX) pathway, the first reaction is abstraction of hydrogen from $\mathrm{C}-10$ of arachidonic acid, which includes reduction of $\mathrm{Fe}^{3+}$ to $\mathrm{Fe}^{2+}$ in enzyme active site. This results in the formation of arachidonic acid radical, which than reacts with oxygen and generates 12-hydroperoxyeicosatetraenoic acid (12-HPETE). Finally, formation of 12(S)-hydroxy-(5Z,8Z,10E,14Z)-eicosatetraenoic acid (12-HETE) is catalysed by glutathione peroxidase (GPx), whereby glutathione (GSH) is oxidised to GS-SG (Figure 3.) [14, 15]. 

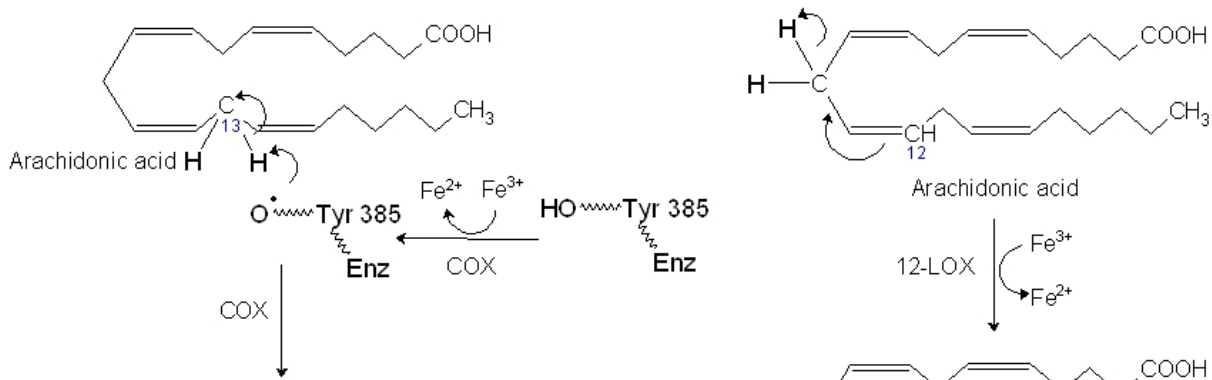

Arachidonic acid

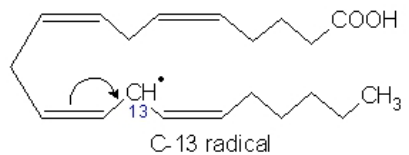

12-LOX $\underset{\mathrm{Fe}^{2+}}{\mathrm{Fe}^{3+}}$
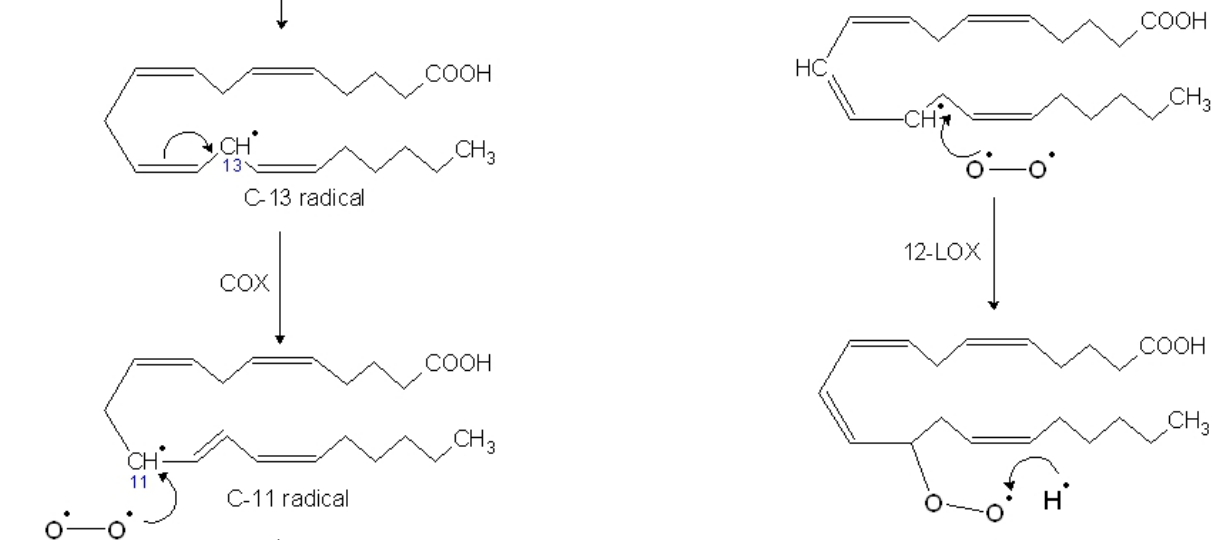

12-LOX
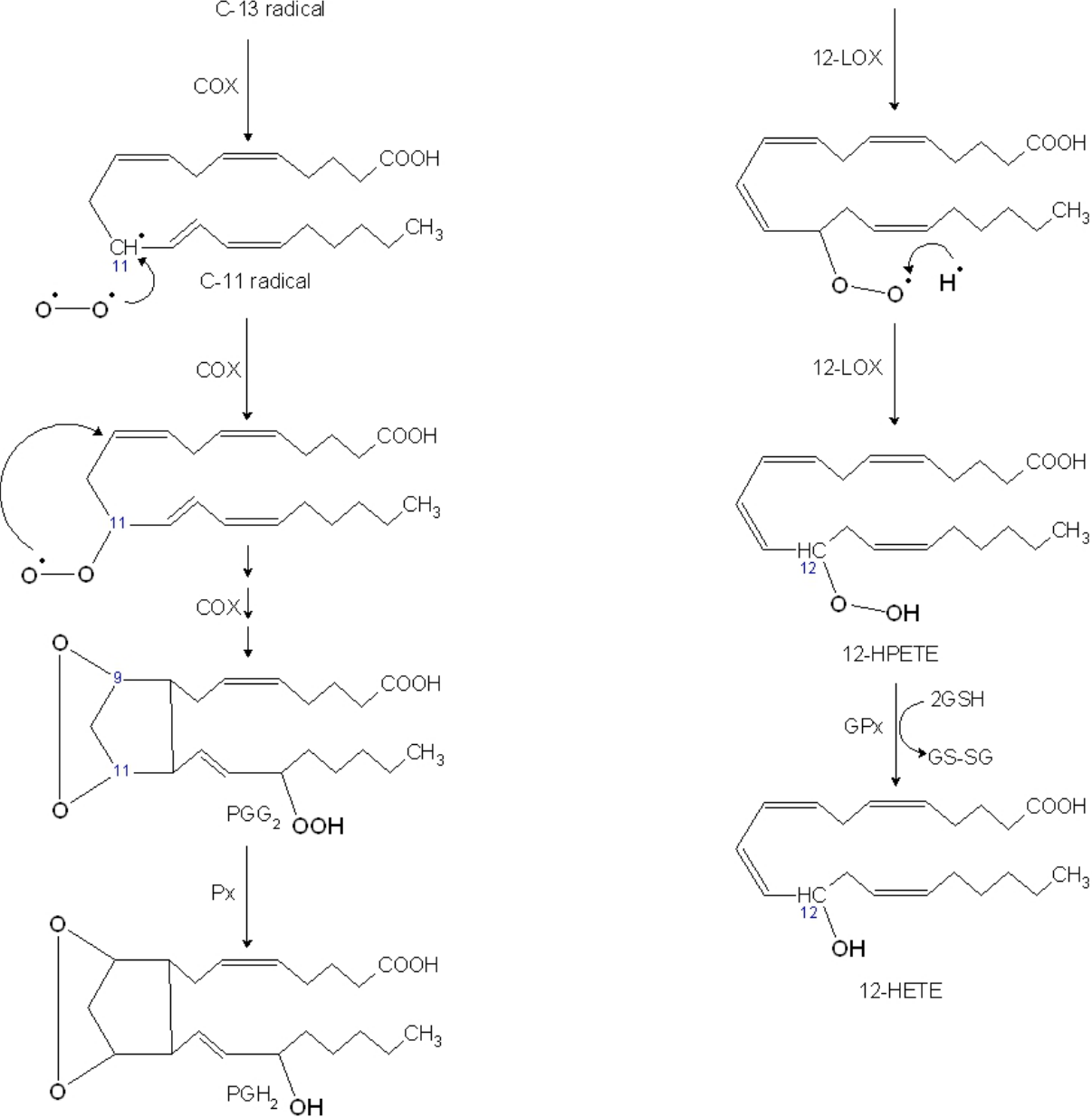

12-HPETE

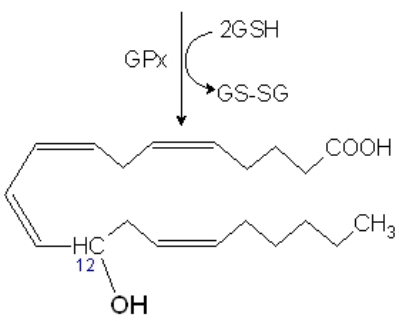

12-HETE

Figure 3. Mechanism of COX and 12-LOX-catalysed arachidonic acid transformation. 
12-HETE is implicated in regulation of platelet aggregation, angiogenesis, as well as the progression of several human diseases like various cancers, rheumatoid arthritis and psoriasis [13, 16, 17]. Also, 12-HETE is known to take part in the metastatic cascade as a crucial intracellular signalling molecule which activates protein kinase $C$ and mediates the biological functions of growth factors and cytokines.

Nowadays, there is a great need for a new anti-inflammatory compounds with minimal side effects. Natural products, especially phenolics frequently consumed in a diet of plant origin, are known to have great anti-inflammatory potential considering inhibition of COX and LOX enzymes $[18,19]$. Thus, screening of plants for COX/LOX inhibitory activity, followed by effect-guided fractionation, can be a useful tool for discovering new secondary biomolecules with anti-inflammatory potential. Although plant species widely used in traditional medicine may be a good starting point, there is also a vast number of currently unexplored species of unknown composition and activity. Therefore, this study included the poorly investigated plant species classified into four families - Alliaceae, Cupressaceae, Plantaginaceae and Polygonaceae - wild growing in Serbia, that are part of our continuing research [20-29]. Most of these species are used in diet or used in folk medicine for healing various disorders.

Considering the fact that lipid peroxidation is involved in an inflammation process, the aim of this study was to compare antioxidant activity (more specifically, the ability to inhibit lipid peroxidation) of the selected plant extracts with their ability to inhibit production of particular arachidonic acid metabolites and to correlate these activities with the phenolic and flavonoid content.

\section{Methods}

\subsection{Plant material and extract preparation}

Plant material used in this study was collected from different locations in Serbia during the period between 2008-2010, during a flowering phase or as ripe specimens. Species belonging to four families: Alliaceae (genus Allium - A. flavum L., A. carinatum L., A. melanantherum Panč., A. pallens L., A. rhodopeum Velen., A. paniculatum L.), Cupressaceae (genus Juniperus L. J. communis L., J. sibirica Burgsdorf., J. foetidissima Willd.), Plantaginaceae (genus Plantago L. - P. major L., P. maritima L., P. media L., P. lanceolata L., P. altisima L.) and Polygonaceae (genus Rumex L. - R. patientia L., R. crispus L., R. obtusifolius L.) were investigated. The voucher specimens were deposited in the Herbarium of the Department of Biology and Ecology (BUNS Herbarium), University of Novi Sad Faculty of Sciences.

Air-dried and grounded plant material (30 g) (whole plants of Allium sp., needles and cones of Juniperus sp., aerial parts of Plantago sp. and herbs and rhizomes of Rumex sp.) was extracted by maceration with $80 \%$ aqueous methanol ( $8 \mathrm{~mL}$ per $1 \mathrm{~g}$ of drug) during $72 \mathrm{~h}$ at room temperature. After filtration, the solvent was evaporated to dryness under reduced pressure. All raw extracts except those of Allium sp. and of Rumex sp. rhizomes were resuspended in hot distilled water (to a final concentration of approx. $1 \mathrm{~g} / \mathrm{mL}$ ), washed 
exhaustively with petroleum ether (fraction $40-60{ }^{\circ} \mathrm{C}$ ) to remove nonpolar pigments, and evaporated to dryness under vacuum.

Dried extracts were dissolved in $80 \%$ aqueous methanol and DMSO for evaluation of the antioxidant and anti-inflammatory activity, respectively, to obtain $300 \mathrm{mg} / \mathrm{mL}$ stock solutions.

\subsection{Determination of total phenolic content}

Total phenolic content was determined according to method of Singleton et al. [30], modified for 96-well microplates. Gallic acid was used as a standard for calibration curve construction. Thirty microliters of each extract or standard solution was added to $150 \mu \mathrm{L}$ of $0.1 \mathrm{~mol} / \mathrm{L}$ Folin-Ciocalteu reagent and after $10 \mathrm{~min}$ mixed with $120 \mu \mathrm{L}$ of sodium carbonate (7.5\%). The same mixture, with solvent instead of extract, was used as a blank. Absorbance at $760 \mathrm{~nm}$ was read after $2 \mathrm{~h}$. The phenolics concentration was determined by using the calibration curve of gallic acid. The total phenolics value was expressed as milligrams of gallic acid equivalents per gram of dry weight $(\mathrm{dw})$.

\subsection{Determination of total flavonoid content}

The aluminium chloride spectrophotometric method described by Chang et al. [31] and modified for 96-well microplates, was used for determination of of the total flavonoid content. Quercetin was used as a standard to prepare a calibration curve. The reaction mixture was comprised of $30 \mu \mathrm{L}$ of the extract or standard solution, $90 \mu \mathrm{L}$ of methanol, $6 \mu \mathrm{L}$ of $10 \%$ aluminium chloride (substituted with distilled water in blank probe), $6 \mu \mathrm{L}$ of $1 \mathrm{~mol} / \mathrm{L}$ potassium acetate and $170 \mu \mathrm{L}$ of distilled water. Absorbance at $415 \mathrm{~nm}$ was measured after $30 \mathrm{~min}$. Flavonoid content was calculated according to the standard calibration curve and are expressed in milligrams of quercetin equivalents per gram of $\mathrm{dw}$.

\subsection{Lipid peroxidation}

There are several methods for measuring the ability of plant extracts to inhibit lipid peroxidation [8]. Some of the methods are based on monitoring of malondialdehyde (MDA), a degradation product of polyunsaturated fatty acids peroxidation. Possible ways for quantification of MDA are GC-FID after derivatisation, HPLC with DAD or fluorimetric detector and spectrophotometric method. The latter is based on the reaction of MDA with thiobarbituric acid (TBA) and it is commonly used both in in vitro and in vivo studies. Formation of the red-coloured MDA-TBA adduct is measured at $532 \mathrm{~nm}$. Different substrates can be used in this test: lecithin liposomes, free fatty acids, LDL and body fluids $[8,32]$. Also, a few different initiators of LP, such as ionizing radiation, chemical agents metal ions, free radicals and metalloproteins may be used [8].

In this study, we used spectrophotometric TBA assay [28, 33] for evaluation the ability of extracts to inhibit LP. Linseed oil, used as a source of polyunsaturated fatty acids $(69.7 \%$ linolenic, $13.5 \%$ linoleic acid, as determined by GC-MS), was obtained from linseed by 
Soxhlet extraction. Oil was added to $0.067 \mathrm{~mol} / \mathrm{L}$ phosphate buffer, $\mathrm{pH} 7.4$, in the presence of $0.25 \%$ Tween- 80 to obtain a $0.035 \%$ suspension and sonicated for 1 hour. This suspension (3.0 mL) was mixed with $20 \mu \mathrm{L}$ of $\mathrm{FeSO}_{4}(4.58 \mathrm{mmol} / \mathrm{L}), 20 \mu \mathrm{L}$ of ascorbic acid $(87 \mu \mathrm{mol} / \mathrm{L})$, and $20 \mu \mathrm{L}$ of extract (or solvent in control); $3.0 \mathrm{~mL}$ of phosphate buffer and $20 \mu \mathrm{L}$ of extract were added in the blank probe. After incubation at $37^{\circ} \mathrm{C}$ for 1 hour, $0.2 \mathrm{~mL} 3.72 \%$ EDTA was added to all samples followed by $2 \mathrm{~mL}$ of an aqueous mixture containing TBA (3.75 $\mathrm{mg} / \mathrm{mL}), \mathrm{HClO}_{4}(1.3 \%)$, and trichloroacetic acid $(0.15 \mathrm{~g} / \mathrm{mL})$. Reaction mixtures were heated at $100{ }^{\circ} \mathrm{C}$ for $15 \mathrm{~min}$, cooled, centrifuged at $1600 \mathrm{~g}$ for $15 \mathrm{~min}$, and absorbance was measured at $532 \mathrm{~nm}$. All samples and control were made in triplicate. IC 50 values were determined from inhibition vs. concentration plots.

\subsection{Anti-inflammatory activity}

There are a great number of different in vitro methods used for estimation of inhibitory activity of COX and LOX enzymes including a number of commercial kits. Some assays, as a source of enzymatic activity, include native or recombinant enzymes, animals or human origin, while others use different cell lines that express desirable activities. Arachidonic acid is often added in reaction medium and in some cases is radio labelled. Induction of inflammatory respond also differs, and in most cases is performed by bacterial lipopolysaccharide, various cytokines and tumour necrosis factor. Different techniques are used for detection of enzymatic activity, such as different chromatographic techniques (TLC, HPLC-UV), as well as EIA $[19,34]$.

In this study, COX-1 and 12-LOX inhibitory activity was investigated using ex vivo assay according to modified method of Safayhi et al. [29, 35]. Intact cells (human platelets) were used as a source of COX-1 and 12-LOX enzymes. Arachidonic acid metabolites (12-HHT and 12-HETE) were determined by use of LC-MS/MS technique [29].

An aliquot of human platelet concentrate (viable but outdated for medical use) containing $4 \cdot 10^{8}$ cells was suspended in buffer $\left(0.137 \mathrm{~mol} / \mathrm{L} \mathrm{NaCl}, 2.7 \mathrm{mmol} / \mathrm{L} \mathrm{KCl}, 2.0 \mathrm{mmol} / \mathrm{L} \mathrm{KH}_{2} \mathrm{PO}_{4}\right.$, $5.0 \mathrm{mmol} / \mathrm{L} \mathrm{Na}_{2} \mathrm{HPO}_{4}$ and $5.0 \mathrm{mmol} / \mathrm{L}$ glucose, $\mathrm{pH}$ 7.2) to obtain final volume of $2 \mathrm{~mL}$. This mixture was slowly stirred at $37^{\circ} \mathrm{C}$ for $5 \mathrm{~min}$. Subsequently, $0.1 \mathrm{~mL}$ of extracts or standard compounds solutions in DMSO (concentration ranging from 10.0 to 200.0, 0.156 to 5.0 and 0.01 to $0.6 \mathrm{mg} / \mathrm{ml}$ for extracts, quercetin and aspirin, respectively) and $0.1 \mathrm{~mL}$ of calcimycin (Calcium Ionophore A23187, $125 \mu \mathrm{mol} / \mathrm{L}$ in DMSO) were added and incubated for $2 \mathrm{~min}$ at $37{ }^{\circ} \mathrm{C}$, with moderate shaking. The exact volume of extract in control and calcimycin in blank probe were substituted with solvent (DMSO). Thereafter, $0.3 \mathrm{~mL}$ of $\mathrm{CaCl}_{2}$ aqueous solution $(16.7 \mathrm{mmol} / \mathrm{L})$, substituted with water in blank probe, was added and the mixture was incubated for further $5 \mathrm{~min}$ at $37^{\circ} \mathrm{C}$ with shaking. Acidification with cold $1 \%$ aqueous formic acid $(5.8 \mathrm{~mL})$ to $\mathrm{pH} 3$ terminated the reaction. If gel formation occurred, vortexing was applied before mixing with the acid. Prostaglandin $\mathrm{B}_{2}(50 \mu \mathrm{L}$ of $6 \mu \mathrm{g} / \mathrm{mL}$ solution in DMSO) was added as internal standard, and extraction of products was done with mixture of chloroform and methanol $(1: 1,8.0 \mathrm{~mL})$ with vigorous vortexing for $15 \mathrm{~min}$. After centrifugation at $7012 \times \mathrm{g}$ for $15 \mathrm{~min}$ at $4{ }^{\circ} \mathrm{C}$, organic layer was separated, evaporated to 
dryness, dissolved in methanol $(0.5 \mathrm{~mL})$, filtered and used for further LC-MS/MS analysis. All samples and control were made in triplicate.

Test for estimation of the anti-inflammatory activity, applied in our research, has a lot of advantages. Firstly, the advantage is avoidance of the undesirable in vivo tests on experimental animals. Even though the exact anti-inflammatory activity can be validated only through in vivo tests, creating in vitro assays, in which physiological conditions similar to in vivo assays are used, can provide valuable information about inhibitory potential of the compounds tested. Platelets are a suitable cell system for testing inhibitory activity, because they can provide physiological cell conditions and possibility to examine the inhibition of both enzymes at the same time. Secondly, for determination of the formed metabolites as a measure of level of inhibition of COX and LOX activity, LC-MS/MS technique was used. LC-MS/MS provided a highly sensitive and specific detection of desirable metabolites within a short analysis time [29].

\subsection{Statistical analysis}

Percent of lipid peroxidation inhibition achieved by different concentration of extracts was calculated by the following equation: $I(\%)=\left(A_{0}-A\right) / A_{0} \times 100$, where $A_{0}$ was the absorbance of the control reaction and $A$ was the absorbance of the examined samples, corrected for the value of blank probe. Percent of COX-1 and 12-LOX inhibition achieved by different concentrations of extracts was calculated by the following equation: $I(\%)=100 \times\left(R_{0}-R\right) / R_{0}$, where $R_{0}$ and $R$ were response ratios (metabolite peak area divided by internal standard peak area) in the control reaction and in the examined samples, respectively. Both $R$ and $R_{0}$ were corrected for the value of blank probe. For both assays, corresponding inhibitionconcentration curves were drawn using Origin software, version 8.0 (Origin Labs) and IC 50 values (concentration of extract that inhibited lipid peroxidation and COX-1 and 12-LOX metabolites formation by $50 \%$ ) were determined. All of the results were expressed as mean \pm SD of three replicates. Correlation analyses were done using Statistica software version 6 (StatSoft). Concentrations of total phenolics and the total flavonoids were used as independent variables, while inhibitory activities towards LP and 12-HETE synthesis (expressed as 1/IC50) were used as dependent variables. Due to a wide range of values, loglog plots were applied. Pearson's correlation coefficients were calculated.

\section{Results}

In this study, we tested the effect of 17 taxa from four families (Alliaceae, Cupressaceae, Plantaginaceae and Polygonaceae) on lipid peroxidation and metabolism of arachidonic acid. The total phenolic and flavonoid contents were determined in the plant extracts, as well. All results are presented in Figures 4, 5, 6 and 9.

Among all tested samples, herb and rhizome extracts of Rumex species were the most potent inhibitors of LP $(0.009-0.047 \mathrm{mg} / \mathrm{mL})$. Slightly lower activity was shown by herb extracts of Plantago species $(0.025-0.178 \mathrm{mg} / \mathrm{mL})$, while extracts of cones and needles of Juniperus species, as well as the whole plant extracts of Allium species expressed a much lower activity (0.117-0.887 and 0.68-1.986 mg/mL, respectively). 


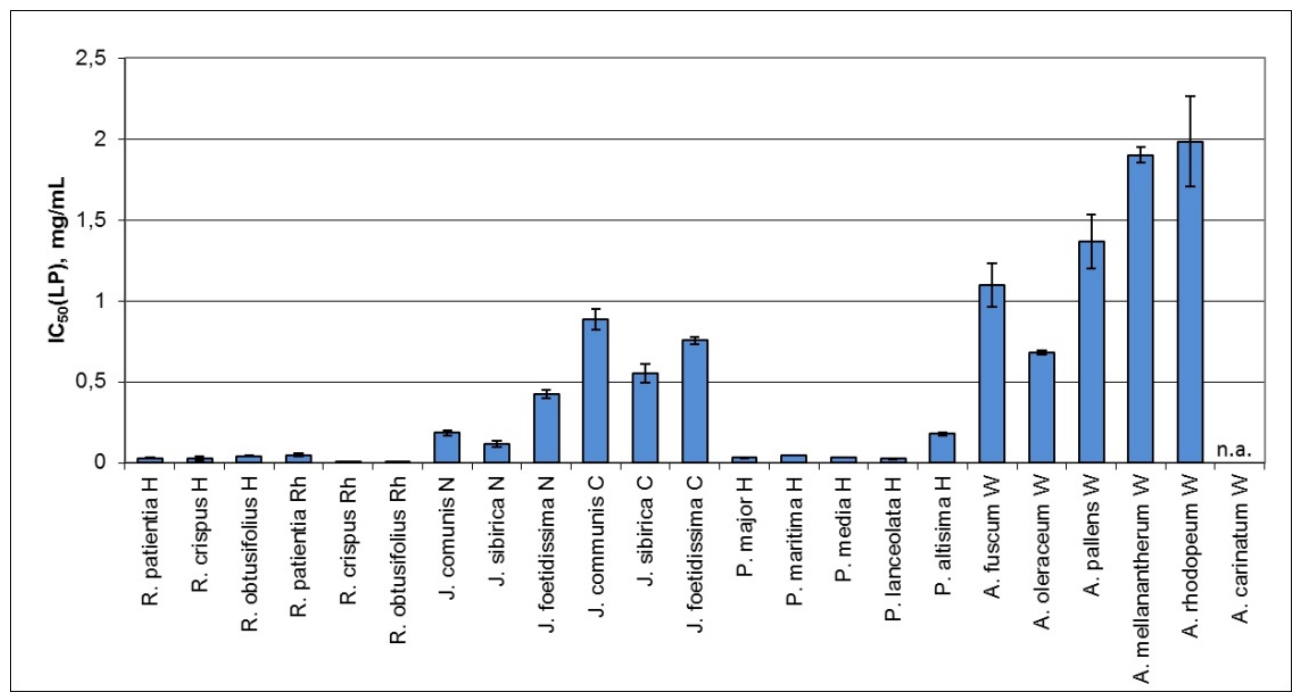

Legend: $\mathrm{H}$ - herb, Rh - rhizome, C - cones, $\mathrm{N}$ - needles, $\mathrm{W}$ - whole plant, n.a. - not achieved.

Figure 4. Results of lipid peroxidation inhibition assay.

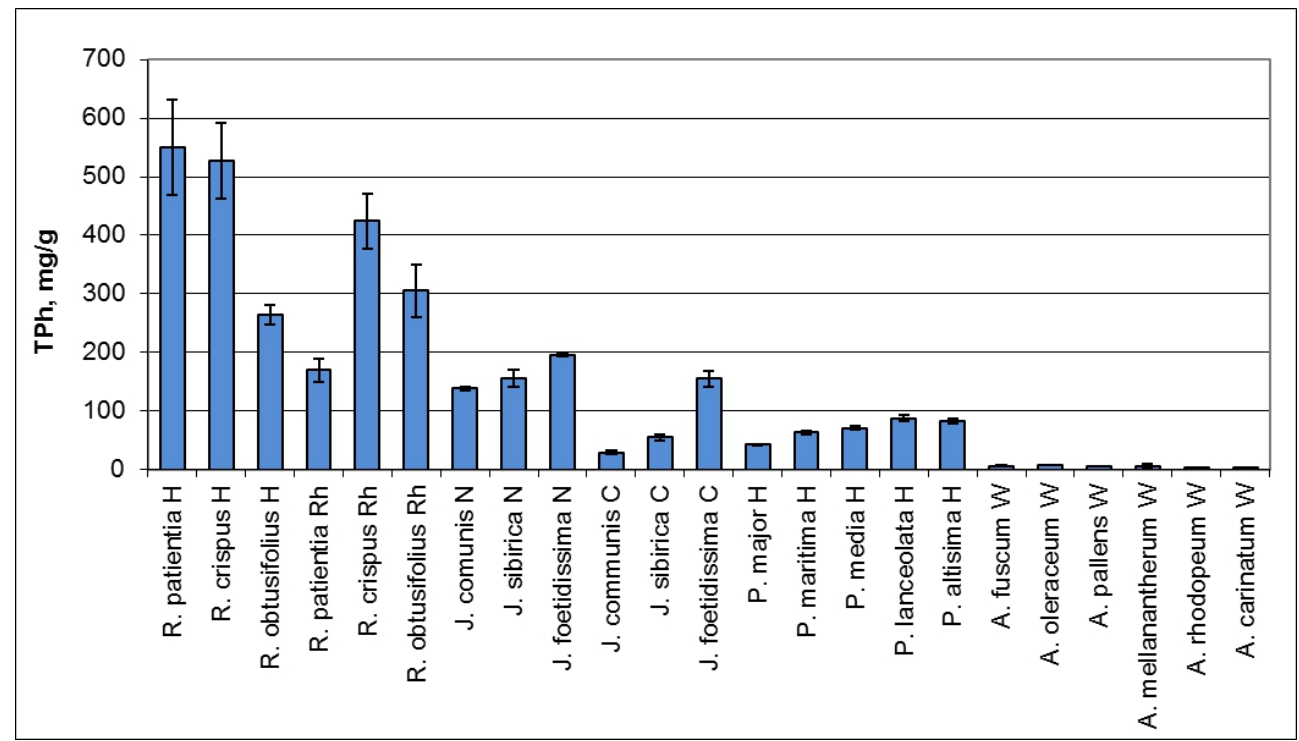

Legend: $\mathrm{H}$ - herb, $\mathrm{Rh}$ - rhizome, $\mathrm{C}$ - cones, $\mathrm{N}$ - needles, $\mathrm{W}$ - whole plant.

Figure 5. Results of total phenolic content assay (expressed as mg eq. gallic acid / $1 \mathrm{~g} \mathrm{dw}$ ).

Total phenolic content in examined genera decreased in following order: Rumex, Juniperus, Plantago and Allium, with R. patentia and R. crispus extracts being by far the richest in phenolics (550 and $527 \mathrm{mg}$ eq. gallic acid per $1 \mathrm{~g} \mathrm{dw}$, respectively). Regarding the content of the total flavonoids, significant intrageneric variations were observed, hence it was not 
possible to point out a genus with the highest content. Flavonoids were the most abundant in J. foetidissima needles extract (60 mg quercetin eq per $1 \mathrm{~g} \mathrm{dw}$ ), and scarcest in A. rhodopeum (0.2 $\mathrm{mg}$ quercetin eq per $1 \mathrm{~g} \mathrm{dw}$ ).

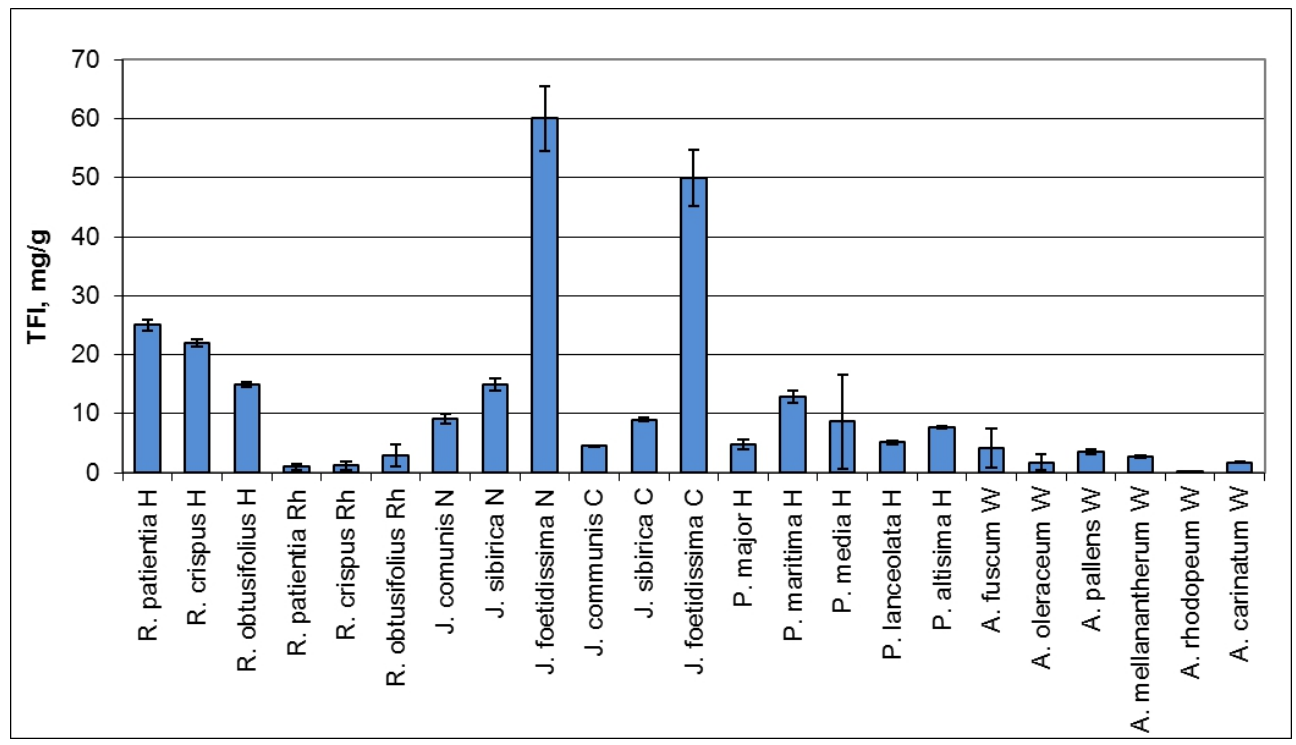

Legend: $\mathrm{H}$ - herb, $\mathrm{Rh}$ - rhizome, $\mathrm{C}$ - cones, $\mathrm{N}$ - needles, $\mathrm{W}$ - whole plant.

Figure 6. Results of total flavonoid content assay (expressed as mg eq. quercetin / $1 \mathrm{~g} \mathrm{dw}$ ).

Plant phenolics present in extracts can counteract lipid peroxidation in either initiation or propagation step. Possible mechanisms are chelation of transition metals $\left(\mathrm{Fe}^{2+}\right)$, reduction of $\mathrm{Fe}^{3+}$ and neutralisation of lipid peroxidation radical intermediates by transfer of hydrogen or single electron [8]. An attempt was made to correlate the observed lipid peroxidation inhibitory activity (given as $1 / \mathrm{IC}_{50}$ ) with the content of the total phenolics and total flavonoids. The corresponding plots are given in Figure 7. and Figure 8.

A high degree of correlation was observed between the lipid peroxidation inhibitory activity and the total phenolic content $(r=0.7713)$, while correlation with the total flavonoids content was not established $(r=0.1410)$. Thus, flavonoids do not contribute to the total observed activity to a significant extent. The lower activity of flavonoids, compared to other phenolics, can be explained through Porter's polar paradox. Namely, flavonoids present in the extracts investigated were predominantly in glycosylated form and thus highly polar. It is demonstrated that compounds of lower polarity are more effective in polar reaction media, such as oil-in-water emulsion used in our experiments, since they exhibit their activity at oil-water interface [36]. In addition, hydrogen atom donation ability of flavonoids decrease with glycosylation, especially if the most active hydroxyl groups (C-3, C-3' or C-4') are occupied by carbohydrate moiety [37, 38].

Regarding the eicosanoid pathway, Rumex and Plantago species showed, on average, very high 12-LOX inhibitory effect, with $\mathrm{IC}_{50}$ in range $0.75-3.59 \mathrm{mg} / \mathrm{mL}$ (Figure 9.). While 
Juniperus and Allium also exhibited dose-dependent inhibition of 12-HETE production, their IC 50 values were higher, ranging from $1.45 \mathrm{mg} / \mathrm{mL}$ to $11.04 \mathrm{mg} / \mathrm{mL}$.

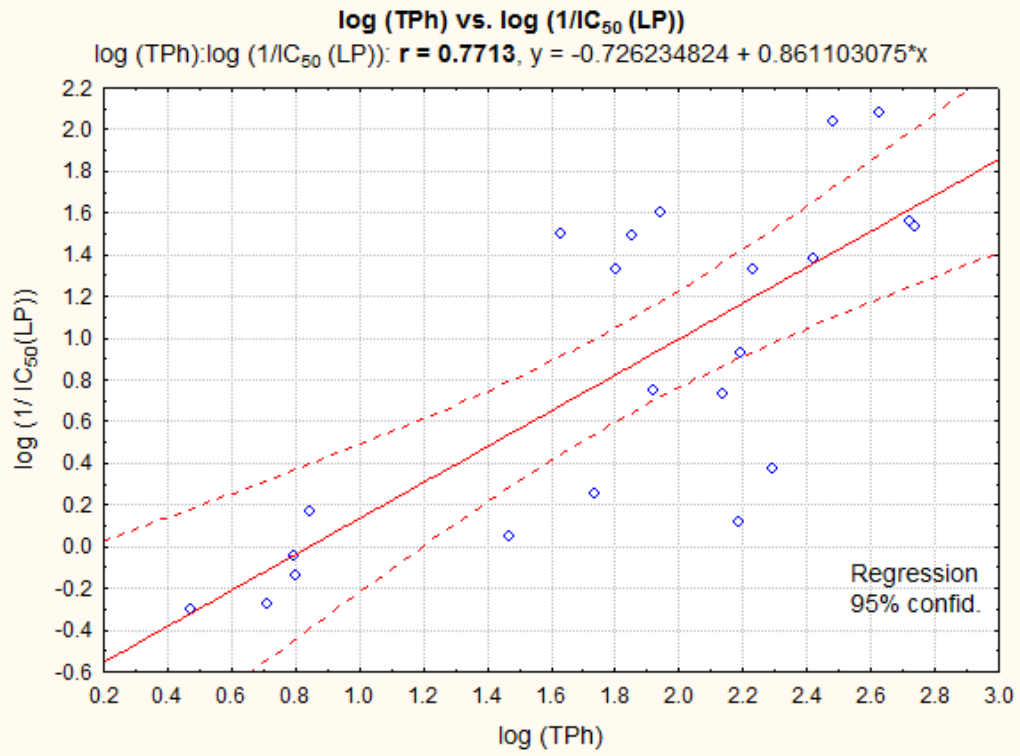

Figure 7. Correlation between total phenolic content and ability of extracts to inhibit lipid peroxidation

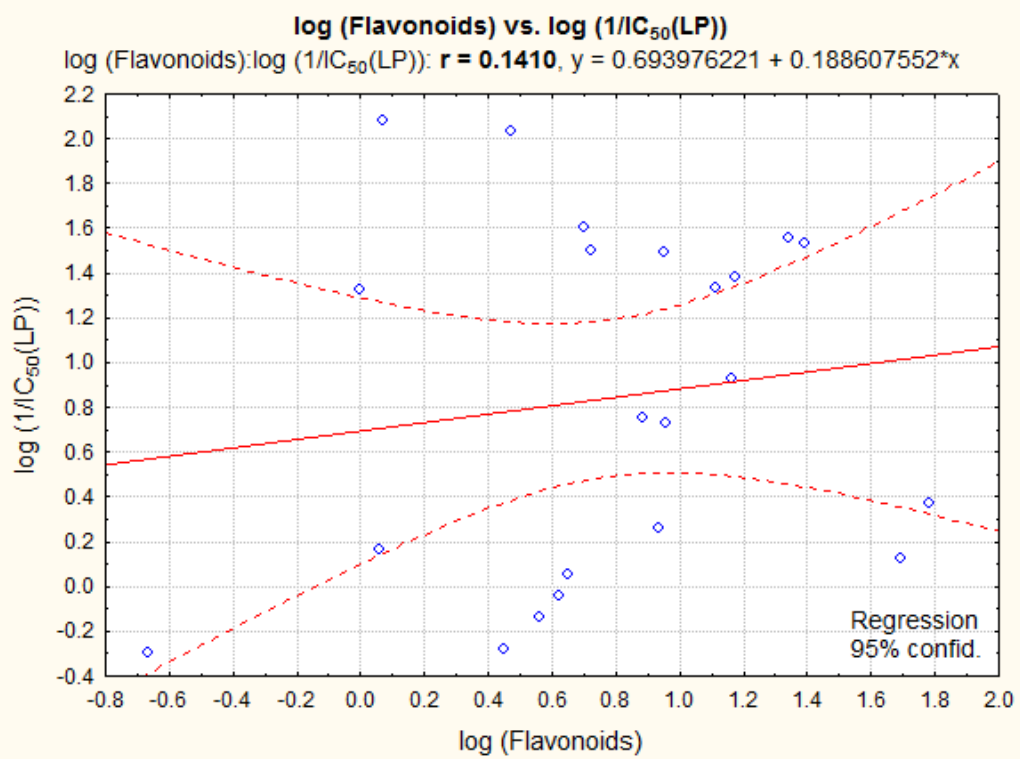

Figure 8. Correlation between total flavonoids content and ability of extracts to inhibit lipid peroxidation 


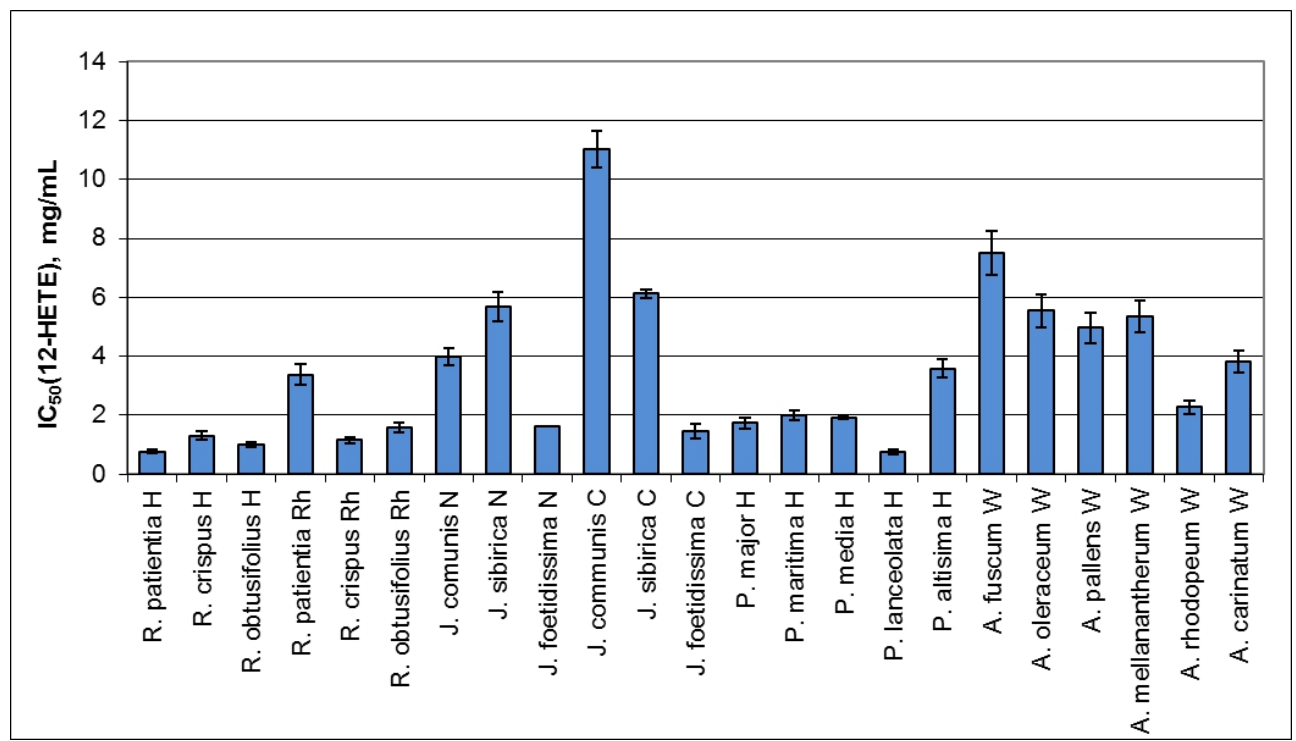

Legend: $\mathrm{H}$ - herb, $\mathrm{Rh}$ - rhizome, $\mathrm{C}$ - cones, $\mathrm{N}$ - needles, $\mathrm{W}$ - whole plant.

Figure 9. Results of 12-LOX inhibition assay.

Moreover, certain inhibitory activity of the examined extracts towards COX-1 was also confirmed in the anti-inflammatory assay applied in this research. Determined IC50 values ranged from 0.34 to $8.00 \mathrm{mg} / \mathrm{mL}$, with no genus exhibiting significantly higher activity than others. However, the results are not shown due to lack of correlation with the total phenolics and flavonoid content. This is in agreement with our previous findings [27].

The exact mechanism of COX and LOX enzymes inhibition by natural products is still not fully elucidated. However, bearing in mind their structures and chemical properties, several mechanisms could be speculated. Both pathways of arachidonic acid metabolism include free radical reactions (Figure 3.). Due to their radical scavenging activity or reducing properties, many plant natural products can interfere with reactions catalysed by COX and LOX [39]. They can neutralise radical intermediates, thus terminating the reaction. In addition, they can reduce $\mathrm{Fe}^{3+}$ ion that is a part of active site of both enzymes and is necessary for initiation reaction. Some natural products, including acetylenes, can bind covalently to enzymes and inhibit them irreversibly [19]. Also, in inhibition of LOX, isoprenyl moiety of some phenolics and terpenoid backbone structure could play an important role. Prenylated phenolics are usually more hydrophobic than conventional ones. Terpenoids are also mostly non-polar compounds. These characteristics suggest an easy penetration through the cell membrane and their good 12-lipoxygenase inhibitory properties [19]. Finally, the COX or LOX activity can be decreased by suppressing their transcription by phenolics [18], although this effect is observable only in long-duration experiments.

To identify the compound class responsible for the observed 12-LOX-inhibitory activity, 1/IC50 was correlated with the content of the total phenolics and flavonoids. The corresponding plots 
are given in Figure 10. and Figure 11. As with the lipid peroxidation, the correlation between the content of the total phenolics and 12-LOX-inhibitory activity was also observed, although slightly weaker, with Pearson's correlation coefficient $r=0.6037$. At the same time, no relationship was found between the total flavonoids content and 12-HETE production $(r=$ 0.2825). Bearing in mind a good correlation of 12-LOX inhibition with the phenolic content, and the lack thereof with the flavonoid content, it is possible that only small phenolic molecules, but not the voluminous flavonoid glycosides, can enter 12-LOX active site and exhibit the inhibitory effect there. Thus, the observed differences in the total phenolics and the total flavonoids effects can at least partially be explained by steric hindrance.

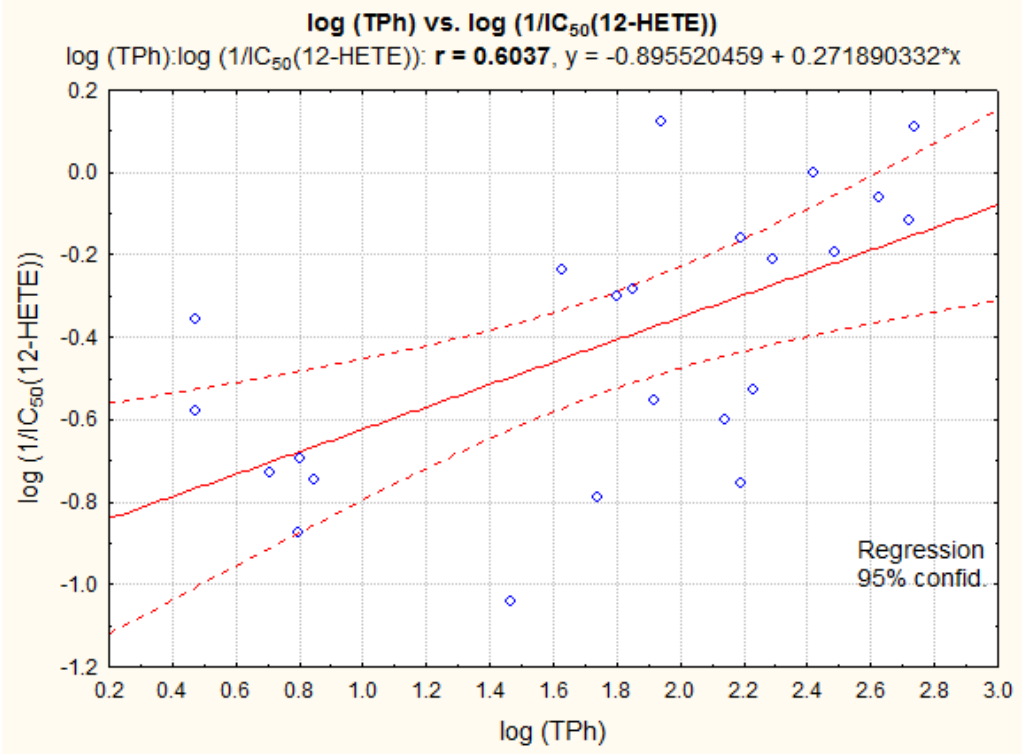

Figure 10. Correlation between total phenolic content and 12-LOX inhibitory activity

The differences in COX and LOX inhibition (LOX inhibition being correlated with phenolic content) can be attributed to the differences in reaction mechanism and active site threedimensional structure. Namely, both mechanisms include abstraction of hydrogen from arachidonic acid leading to formation of radical species. However, hydrogen acceptor in COX is tyrosyl radical (formed through tyrosine oxidation by $\mathrm{Fe}^{3+}$ ) while in $\mathrm{LOX}$, electron is transferred directly to $\mathrm{Fe}^{3+}$. Phenolics from plant extracts could reduce $\mathrm{Fe}^{3+}$ to $\mathrm{Fe}^{2+}$, thus inactivating both enzymes. However, the presence of tyrosyl residue in COX active site could provide steric protection and prevent phenolics from approaching the $\mathrm{Fe}^{3+}$ ion.

Finally, the correlation between the ability of extracts to counteract lipid peroxidation and inhibit production of 12-HETE is shown in Figure 12. High correlation coefficient $(r=0.6819)$ suggests that extracts with a high inhibitory effect on LP, also represent potent inhibitors of 12-LOX pathway. Methods for examination of anti-inflammatory activity are labourintensive, expensive and sometimes involve ethical issues due to the usage of laboratory 
animals. Therefore, in vitro measurement of lipid peroxidation inhibition and the total phenolic content could be a useful tool for screening of plant extracts with a potential antiinflammatory activity.

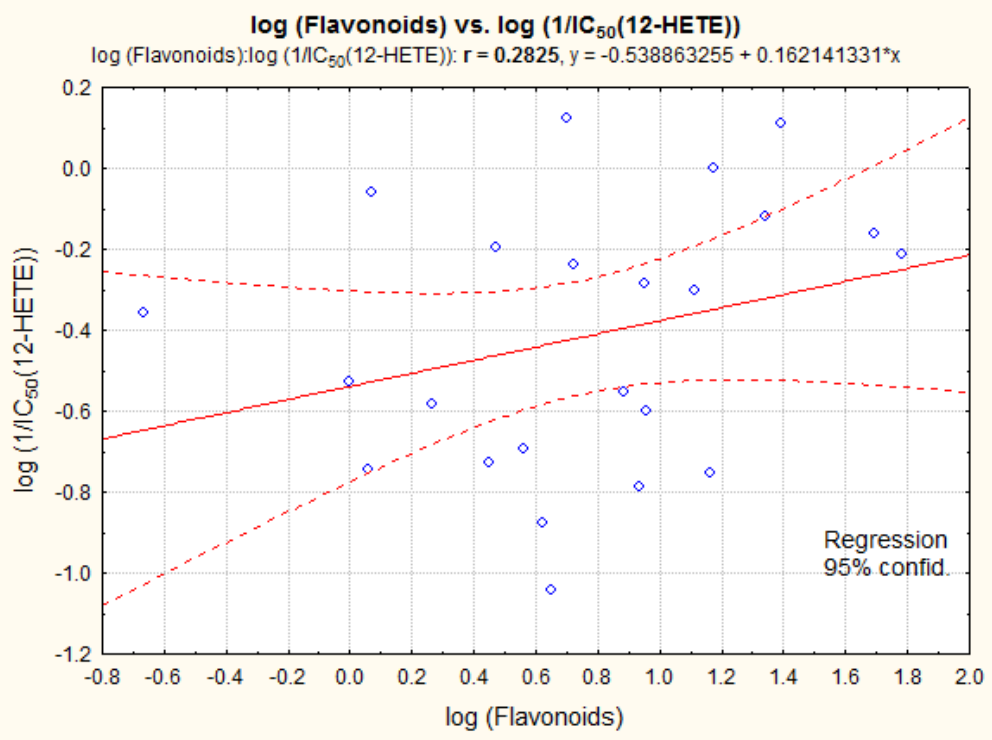

Figure 11. Correlation between total flavonoid content and 12-LOX inhibitory activity

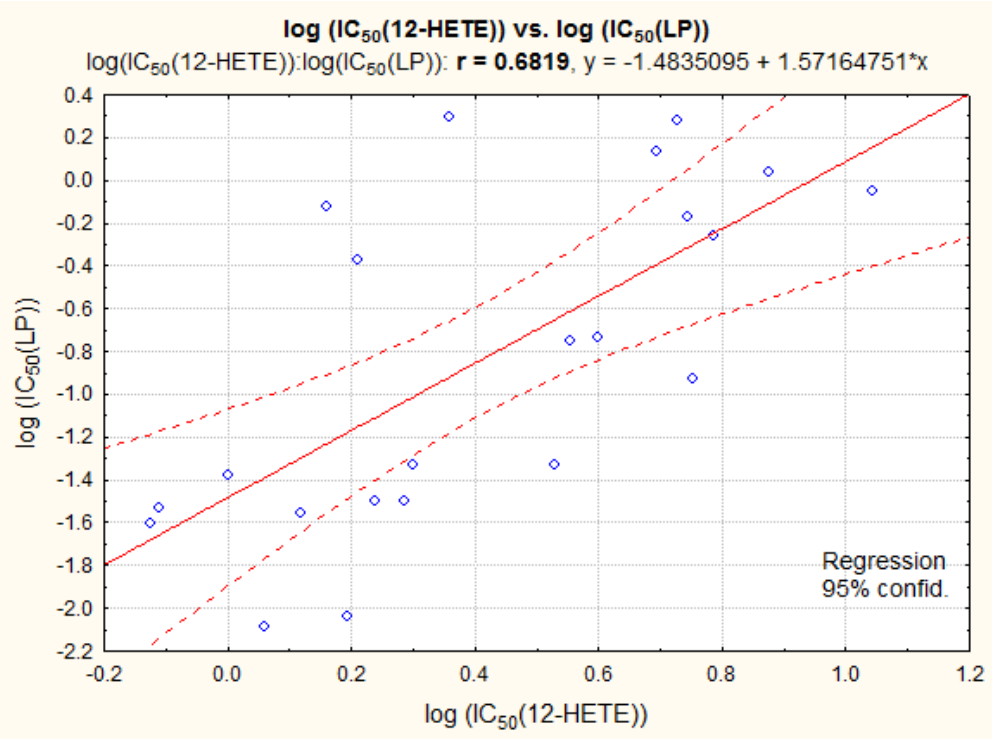

Figure 12. Correlation between the ability of extracts to inhibit 12-HETE production and lipid peroxidation 
To summarise, the most of the examined species expressed high lipid peroxidation and antiinflammatory activity, especially Rumex and Plantago species. High inhibitory activity of these species towards 12-LOX pathway makes them good candidates for further research taking into account role of this enzyme in cancer development. A good correlation was found between total phenolic content of 23 investigated plant extracts and their ability to inhibit lipid peroxidation and 12-LOX pathway. Consequently, LP inhibitory activity was also highly correlated with 12-LOX inhibition. Thus it can serve as indicator for preliminary selection of plant extracts further to be tested for anti-inflammatory activity by expensive and time-consuming methods.

\section{Author details}

Neda Mimica-Dukić, Nataša Simin, Emilija Svirčev, Dejan Orčić, Ivana Beara and Marija Lesjak

Department of Chemistry, Biochemistry and Environmental Protection, University of Novi Sad, Faculty of Sciences, Novi Sad, Republic of Serbia

Biljana Božin

Department of Pharmacy, Faculty of Medicine, University of Novi Sad, Novi Sad, Republic of Serbia

\section{Acknowledgement}

The Ministry of Education and Science, Republic of Serbia (Grant No. 172058) funded this research. We wish to thank to Goran Anačkov, $\mathrm{PhD}$, for the providing voucher specimens, and Gordana Vlahović, MSc, for language corrections. Platelet concentrate was kindly provided by The Institute for Blood Transfusion of Vojvodina, Novi Sad, Republic of Serbia.

\section{References}

[1] Kawanishi S., Hiraku Y., Murata M., Oikawa S. The role of metals in site-specific DNA damage with reference to carcinogenesis. Free Radical Biology \& Medicine 2002;32(9) 822-832.

[2] Manach C., Mazur A., Scalbert A. Polyphenols and prevention of cardiovascular diseases. Current Opinion in Lipidology 2005;16(1) 77-84.

[3] Gonsette RE. Review: Oxidative stress and excitotoxicity: a therapeutic issue in multiple sclerosis? Multiple Sclerosis 2008;14(1) 22-34.

[4] Maritim AC., Sanders RA., Watkins JB. Diabetes, Oxidative Stress, and Antioxidants: A Review. Journal of Biochemical and Molecular Toxicology 2003;17(1) 24-38.

[5] Ramassamy C. Emerging role of polyphenolic compounds in the treatment of neurodegenerative diseases: a review of their intracellular targets. European Journal of Pharmacology 2006;545(1) 51-64.

[6] Wruck CJ., Fragoulis A., Gurzynski A., Brandenburg L., Kan YW., Chan K., Hassenpflug J., Freitag-Wolf S., Varoga D., Lippross S., Pufe T. Role of oxidative stress 
in rheumatoid arthritis: insights from the Nrf2-knockout mice. Annals of the Rheumatic Diseases 2010;doi:10.1136/ard.2010.132720

[7] Alvarado C., Alvarez P., Jimenez L., De la Fuente M. Oxidative stress in leukocytes from young prematurely aging mice is reversed by supplementation with biscuits rich in antioxidants. Developmental \& Comparative Immunology 2006;30(12) 1168-1180.

[8] Laguerre M., Lecomte J., Villeneuve P. Evaluation of the ability of antioxidants to counteract lipid oxidation: Existing methods, new trends and challenges. Progress in Lipid Research 2007;46(5) 244-282.

[9] Gordon MH. The mechanism of antioxidant action in vitro. In: Hudson BJF. (ed.) Food antioxidants. Amsterdam: Elsevier; 1990. p1-18.

[10] Smith WL. The eicosanoids and their biochemical mechanisms of action. Biochemical Journal 1989;259(2) 315-324.

[11] Marnett LJ., Rowlinson SW., Goodwin DC., Kalgutkar AS., Lanzo CA. Arachidonic acid oxygenation by COX-1 and COX-2. Mechanisms of catalysis and inhibition. The Journal of Biological Chemistry 1999;274(33) 22903-22906.

[12] Hawkey CJ. COX-2 inhibitors. The Lancet 1999;353(9149) 307-314.

[13] Greene ER., Huang S., Serhan CN., Panigrahy D. Regulation of inflammation in cancer by eicosanoids. Prostaglandins and Other Lipid Mediators 2011;96(1-4): 27-36.

[14] Yamamoto S., Suzuki H., Ueda N., Takahashi Y., Zoshimoto T. Mammalian Lipoxygenases. In: Curtis-Prior PB. (ed.) The Eicosanoids. Cambridge: Wiley; 2004. p5361.

[15] Müller-Decker K. Cyclooxygenases. In: Marks F., Fürstenberger G. (ed.) Prostaglandins, leukotrienes, and other eicosanoids: from biogenesis to clinical applications. Weinheim: Wiley-VCH; 1999. p65-83.

[16] Liagre B., Vergne P., Rigaud M., Beneytout JL. Expression of arachidonate platelet-type 12-lipoxygenase in human rheumatoid arthritis type B synoviocytes. FEBS Letters 1997;414(1) 159-164.

[17] Müller K. 5-lipoxygenase and 12-lipoxygenase: attractive targets for the development of novel antipsoriatic drugs. Archiv der Pharmazie. 1994;327(1) 3-19.

[18] Lee JH., Kim GH. Evaluation of Antioxidant and Inhibitory Activities for Different Subclasses Flavonoids on Enzymes for Rheumatoid Arthritis. Journal of Food Science. 2010;75(7) H212-H217.

[19] Schneider I., Bucar F. Lipoxygenase Inhibitors from Natural Plant Sources. Part 2: Medicinal Plants with Inhibitory Activity on Arachidonate 12-lipoxygenase, 15lipoxygenase and Leukotriene Receptor Antagonists. Phytotherapy Research. 2005;19(4) 263-272.

[20] Beara IN., Lesjak MM., Orčić DZ., Simin NĐ., Četojević-Simin DD., Božin BN., MimicaDukić NM. Comparative analysis of phenolic profile, antioxidant, anti-inflammatory and cytotoxic activity of two closely-related Plantain species: Plantago altissima L. and Plantago lanceolata L. LWT - Food Science and Technology. 2012;47(1) 64-70.

[21] Lesjak MM., Beara IN., Orčić DZ., Anačkov GT., Balog KJ., Francišković MM., MimicaDukić NM. Juniperus sibirica Burgsdorf. as a novel source of antioxidant and antiinflammatory agents. Food Chemistry. 2011;124(3) 850-856. 
[22] Orčić DZ., Mimica-Dukić NM., Francišković MM., Petrović SS., Jovin ED. Antioxidant activity relationship of phenolic compounds in Hypericum perforatum L. Chemistry Central Journal. 2011;5(1) 34-41.

[23] Beara I. Phytochemical screening and evaluation of antioxidant and anti-inflammatory potential of secondary metaboliotes of Plantago L. species. PhD thesis. University of Novi Sad; 2010.

[24] Orčić D. Scandiceae tribe (Apiaceae Lindley 1836, subfam. Apioideae) species potential resources of biologically and pharmacologically active secondary biomolecules. PhD thesis. University of Novi Sad; 2010.

[25] Mimica-Dukić NM., Simin NĐ., Cvejić JM., Jovin ED., Orčić DZ., Božin BN. Phenolic compounds in field horsetail (Equisetum arvense L.) as natural antioxidants. Molecules. 2008;13(7) 1455-1464.

[26] Božin BN., Mimica-Dukić NM., Simin NĐ., Anačkov GT. Characterization of the volatile composition of essential oils of some lamiaceae spices and the antimicrobial and antioxidant activities of the entire oils. Journal of Agricultural and Food Chemistry. 2006;54(5) 1822-1828.

[27] Lesjak M. Biopotential and chemical characterization of extracts and essential oils of species from Juniperus L. genus (Cupressaceae). PhD thesis. University of Novi Sad; 2011.

[28] Beara I., Lesjak M., Jovin E., Balog K., Anačkov G., Orčić D., Mimica-Dukić N. Plantain (Plantago L.) Species as novel sources of flavonoid antioxidants. Journal of Agricultural and Food Chemistry. 2009;57(19) 9268-9273.

[29] Beara I., Orčić D., Lesjak M., Mimica-Dukić N., Peković B., Popović M. Liquid chromatography/tandem mass spectrometry study of anti-inflammatory activity of Plantain (Plantago L.) species. Journal of Pharmaceutical and Biomedical Analysis. 2010;52(5):701-706.

[30] Singleton VL., Orthofer R., Ramuela-Raventos RM. Analysis of total phenols and other oxidation substrates and antioxidants by means of Folin-Ciocalteu reagent. Methods in Enzymology. 1999;299 152-178.

[31] Chang CC., Yang MH., Wen HM. Chern JC. Estimation of total flavonoid content in propolis by two complementary colorimetric methods. Journal of Food and Drug Analysis. 2002;10(3) 178-182.

[32] Mavi A., Lawrence GD., Kordalia A., Yildirim A. Inhibition of iron-fructose-phosphateinduced lipid peroxidation in lecithin liposome and linoleic acid emulsion systems by some edible plants. Journal of Food Biochemistry. 2011;35(3) 833-844.

[33] Aust SD. Lipid peroxidation. In: Greenwald RA. (ed.) Handbook of methods for oxygen radical research. Boca Raton: CRC Press, 1985. p203-207.

[34] Pairet M., Ryn van J. Experimental models used to investigate the differential inhibition of cyclooxygenase-1 and cyclooxygenase- 2 by non-steroidal anti-inflammatory drugs. Inflammation Research. 1998;47(2) S93-101.

[35] Safayhi H., Mack T., Sabieraj J., Anazodo MI., Subramanian LR., Ammon HPT. Boswellic acids: novel, specific, nonredox inhibitors of 5-lipoxygenase. Journal of Pharmacology and Experimental Therapeutics. 1992;261(3) 1143-1146. 
[36] Porter WL. Paradoxical behavior of antioxidants in food and biological systems. Toxicology and Industrial Health. 1993;9(1-2) 93-122.

[37] Rice-Evans CA., Miller NJ., Paganga G. Structure-antioxidant activity relationships of flavonoids and phenolic acids. Free Radical Biology and Medicine. 1996;20(7) 933-56.

[38] Rice-Evans CA., Miller NJ., Paganga G. Antioxidant properties of phenolic compounds. Trends in Plant Science. 1997;2(4) 152-159.

[39] Hostettmann K. Assays for immunomodulation and effects on mediators of inflammation. In: Dey PM., Harborne JB. (ed.) Methods in Plant Biochemistry vol. 6, London: Academic Press Limited; 1991. p207-211. 